\title{
High infiltration of CD68+ macrophages is associated with poor prognoses of head and neck squamous cell carcinoma patients and is influenced by human papillomavirus
}

Imelda Seminerio ${ }^{1}$, Nadège Kindt ${ }^{1}$, Géraldine Descamps ${ }^{1}$, Justine Bellier ${ }^{1,5}$, Jérôme R. Lechien', Quentin Mat ${ }^{1}$, Charles Pottier ${ }^{2}$, Fabrice Journé ${ }^{1,3}$ and Sven Saussez ${ }^{1,4}$

\footnotetext{
${ }^{1}$ Department of Human Anatomy and Experimental Oncology, Research Institute for Health Sciences and Technology, Faculty of Medicine and Pharmacy, University of Mons, Mons B-7000, Belgium

${ }^{2}$ Department of Pathology, C.H.U., SART TILMAN, University of Liège, Liège 4000, Belgium

${ }^{3}$ Laboratory of Oncology and Experimental Surgery, Jules Bordet Institute, Université Libre de Bruxelles, Brussels 1000, Belgium

${ }^{4}$ Department of Oto-Rhino-Laryngology, Faculty of Medicine, Université Libre de Bruxelles (ULB), Brussels B-1000, Belgium

${ }^{5}$ Present address: Metastasis Research Laboratory, GIGA-Cancer, University of Liege, Liège 4000, Belgium

Correspondence to: Imelda Seminerio, email: imelda.seminerio@umons.ac.be

Sven Saussez, email: sven.saussez@umons.ac.be

Keywords: head and neck squamous cell carcinoma; human papillomavirus; CD68; macrophages; mouse

Received: November 30, $2017 \quad$ Accepted: January 19, $2018 \quad$ Published: January 24, 2018

Copyright: Seminerio et al. This is an open-access article distributed under the terms of the Creative Commons Attribution License 3.0 (CC BY 3.0), which permits unrestricted use, distribution, and reproduction in any medium, provided the original author and source are credited.
}

\section{ABSTRACT}

Incidence of human papillomavirus (HPV)-related head and neck squamous cell carcinomas (HNSCCs) has increased over the last few decades. The reaction of the host immune system to these tumors remains biologically complex. Here, we investigated CD68+ macrophage numbers, reporting the prognostic value in comparison to other risk factors. We also examined CD68+ macrophage infiltration during disease progression regarding the impact of HPV infection, and we studied the role of HPV16-E6/E7 oncoproteins in CD68+ macrophage recruitment. CD68+ macrophage numbers were evaluated in $\mathbf{1 0}$ cases of tumor-free peri-tumoral epithelia, 43 cases of low-grade dysplasia, 45 cases of high-grade dysplasia and 110 cases of carcinoma. Our in vivo model was developed in $80 \mathrm{C} 3 \mathrm{H} / \mathrm{HeN}$ mice orthotopically injected with HPV16-E6, -E7 or -E6/E7-transfected SCC-VII cell lines. High CD68+ macrophage numbers in the intra-tumoral compartment were associated with shorter patient survival (recurrence-free survival: $p=0.001$; overall survival: $p=0.01$ ). Multivariate analyses reported that CD68+ macrophage infiltration and tumor stage were strong and independent prognostic factors of HNSCC. CD68+ macrophage numbers increased during HNSCC progression both in intra-epithelial $(p<0.001)$ and stromal compartments $(p<0.001)$. A higher density of CD68+ macrophages was observed in advanced stages $(p=0.004)$. Patients with transcriptionally active HPV infections had higher CD68+ macrophage density than did HPV-negative patients $(p=0.003)$. CD68+ macrophage infiltration was higher in HPV-E7+ and -E6/E7+ mouse tumors than in $-E 6+$ tumors $(p=0.029$ and $p<0.001)$. In conclusion, the extent of CD68+ macrophage infiltration is a significant prognostic factor for HNSCC patients. The recruitment of macrophages increases during disease progression and is influenced by the HPV virus. 


\section{INTRODUCTION}

Head and neck squamous cell carcinoma (HNSCC) remains one of the most common cancers worldwide, with more than 550,000 new cases diagnosed each year $[1,2]$. Tobacco and alcohol abuse is the main risk factor for these cancers, but infection with oncogenic human papillomavirus (HPV) also appears to be involved in head and neck carcinogenesis [3, 4]. Indeed, we observed an increasing proportion of HPV-infected HNSCCs in nonsmoking and non-drinking young people $(<45$ years old) $[5,6]$. However, there is a discrepancy between this subgroup of patients versus older ( $>45$ years old) smoking and drinking patients in terms of the prognostic value related to HPV infection in HNSCCs. In fact, many studies suggest that HPV-positive HNSCC patients have better overall survival than do HPV-negative patients, suggesting that HPV-infected patients could have better prognoses $[7,8]$. On the other hand, our research lab showed that HPV-positive HNSCC patients have a lower response to concomitant chemoradiotherapy and a decreased 5-year disease-free survival rate than do HPV-negative patients, highlighting their poorer prognoses $[9,10]$. In fact, it appears that the biology of HNSCCs is more complex than we know, underlying that we must consider both HPV status (transcriptionally active or not) and classical risk factors (i.e., tobacco and alcohol consumption) [11].

The host immune system plays a critical role in the development and progression of HNSCCs. Among immune cells, macrophages constitute strong mediators of inflammatory responses, particularly in the fight against cancer $[12,13]$. Depending on the tumor environment stimuli, macrophages present two different phenotypes. Macrophages of the M1 phenotype contribute to cytotoxic CD8+ T cell activation and naïve $\mathrm{CD} 4+\mathrm{T}$ cell differentiation into Th1 effector cells, leading to antitumor effects [14-16]. Among M2 macrophages, tumorassociated macrophages (TAMs) stimulate regulatory $\mathrm{T}$ cell differentiation and secrete several factors (e.g., TGF- $\beta$, TNF- $\alpha$ and IL-10) to create a favorable environment for tumor growth and immunosuppression promotion $[17,18]$.

In squamous cell carcinomas, recent studies found a positive correlation between $\mathrm{CD} 68+$ macrophages (both M1 and M2 phenotypes) and tumor progression in cervical cancers [19-21]. Moreover, a high number of M1 macrophages appears to be an independent prognostic factor for longer survival in patients with cervical carcinoma [22]. Finally, several studies have described a positive correlation between M2 macrophage infiltration and tumor progression of HNSCCs [23].

In the same way, HPV infection plays an important role in tumor progression by modulating the tumor immune environment in order to promote tumor escape. In fact, our previous studies showed that Langerhans cell infiltration is a significant prognostic factor for HNSCCs and that the number of these immune cells is decreased in
HPV-positive HNSCCs [24]. Likewise, we demonstrated increased regulatory T-cell numbers in HPV-related HNSCCs [25, 26]. Moreover, HPV interacts with CD68+ macrophages by recruiting them to the tumor site. Lepique et al. observed a high CD68+ macrophage infiltration rate in an animal model of HPV16-E6/E7-induced tumors, and this macrophage population was mainly constituted of TAMs [27]. Finally, the density of CD68+ macrophages appears to be higher in the tumor area (but not in the stromal regions) of HPV-positive oropharyngeal squamous cell carcinomas (OPSCCs) than in HPV-negative OPSCCs [28].

Here, we evaluated the CD68+ macrophage numbers during HNSCC progression in a large clinical series composed of 10 cases of tumor-free peri-tumoral epithelia (TFE), 43 cases of low-grade dysplasia (LGD), 45 cases of high-grade dysplasia (HGD) and 110 cases of carcinoma (CA), including 74 cases of HPV-negative and 36 cases of HPV-positive HNSCCs. We also assessed the prognostic value of CD68+ macrophage numbers in the stromal and intra-epithelial compartments of these patients, compared to other classical risk factors such as tobacco and alcohol consumption, HPV status and tumor stage. Finally, we conducted in vivo studies to investigate whether HPV16-E6 and -E7 oncoproteins modulate CD68+ macrophage recruitment in an orthotopic mouse model of HNSCC.

\section{RESULTS}

\section{CD68+ macrophage number is associated with poor prognosis in HNSCC}

We assessed the overall survival (OS) rate and the recurrence-free survival (RFS) rate of patients with HNSCC in intra-tumoral and stromal compartments according to CD68+ macrophage numbers. We first used the Cutoff finder web application to estimate the optimal cutoff point for the CD68+ macrophage population; we found that 32 (when evaluating the intra-tumoral compartment) and 67 (when evaluating the stromal compartment) were the optimal cutoff values for CD68+ macrophage numbers. In the intra-tumoral compartment, a high number of CD68+ cells (>32) was statistically associated with a poorer prognosis in terms of RFS (logrank test, $p=0.001$ ) (Figure $1 \mathrm{~A}$ ) and OS of patients with HNSCC (log-rank test, $p=0.01$ ) (Figure 1B). However, no significant correlation between CD68+ macrophage number and prognosis was found regarding OS and RFS in the stromal compartment.

Regarding tumor stage, patients with advanced stages (III-IV) had shorter RFS ( $p=0.006)$ (Figure 1C) and OS $(p=0.005)$ than did other patients (Figure 1D). Finally, multivariate analyses indicated that CD68+ macrophage number ( $p=0.002 \mathrm{RFS} ; p=0.016 \mathrm{OS})$ and tumor stage ( $p=0.014 \mathrm{RFS} ; p=0.014 \mathrm{OS}$ ) had more 
significant prognostic value than did classical risk factors as such as tobacco, alcohol and HPV status (Table 1).

\section{CD68+ macrophages are correlated with tumor stage, tumor location and HPV status}

The association between CD68+ macrophage number and age, gender, tumor location, histological
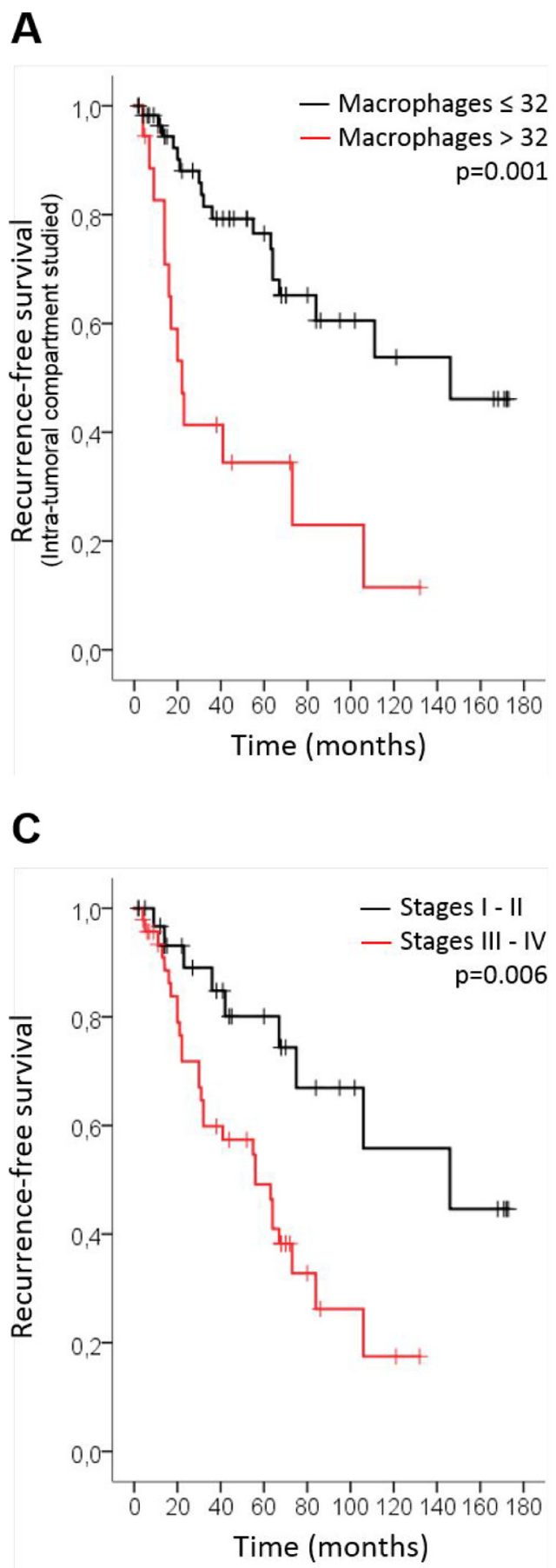

grade, tumor stage, tobacco and alcohol consumption and HPV status was evaluated in both the intra-tumoral and stromal compartments of 110 HNSCC samples (Table 2). No significant correlation was observed regarding CD68+ macrophage number and age, gender, histological grade, tobacco and alcohol consumption in either compartment. However, we noticed a significant correlation between CD68+ macrophages and tumor stage $(p=0.004)$, showing

B

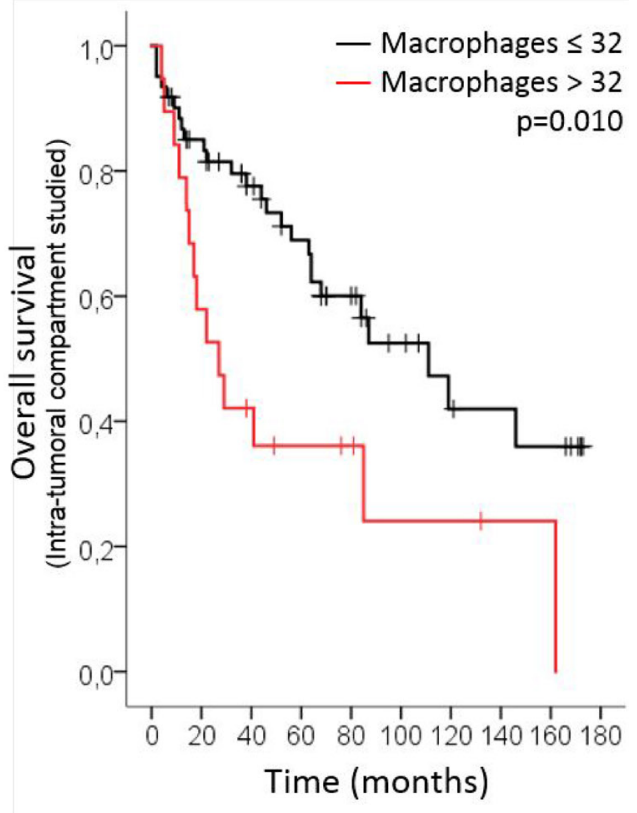

D

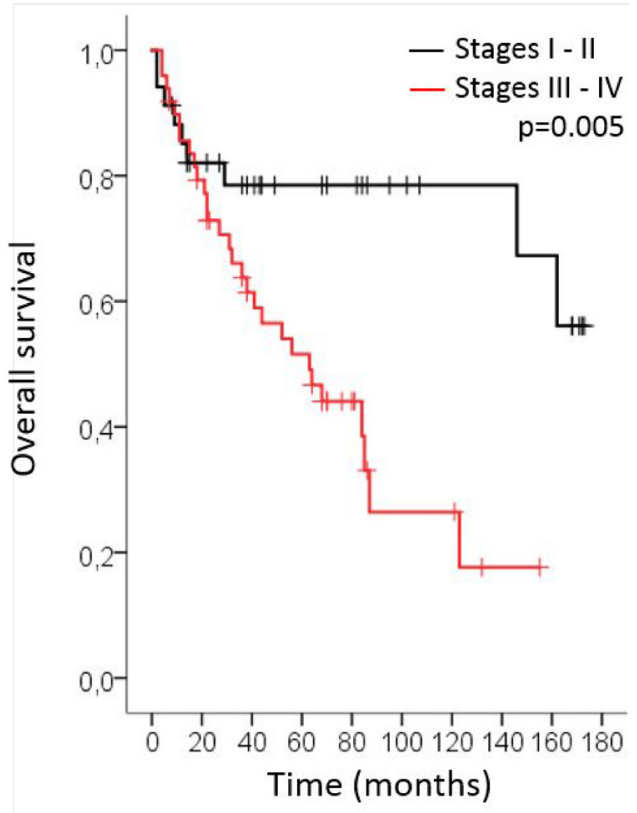

Figure 1: CD68+ macrophage number is associated with poor prognosis in HNSCC. Kaplan-Meier curves of the recurrencefree survival (RFS) (cutoff at 32) (log-rank test, $p=0.001$ ) (A) and overall survival (OS) (cutoff at 32) (log-rank test, $p=0.01)(\mathbf{B})$ of patients with HNSCC in the intra-tumoral compartment according to the number of CD68+ macrophages. Kaplan-Meier curves of the RFS (log-rank test, $p=0.006)(\mathbf{C})$ and OS (log-rank test, $p=0.005)(\mathbf{D})$ according to tumor stage. 
Table 1: Univariate and multivariate Cox regression models evaluating the involvement of CD68+ macrophage number, tobacco, alcohol, tumor stage and HPV status on recurrence-free survival (RFS) and overall survival (OS)

\begin{tabular}{|c|c|c|c|c|}
\hline \multirow[b]{2}{*}{ Variables } & \multicolumn{2}{|c|}{ RFS } & \multicolumn{2}{|l|}{ OS } \\
\hline & HR $(95 \% \mathrm{CI})$ & $p$ value & HR $(95 \%$ CI) & $p$ value \\
\hline \multicolumn{5}{|l|}{ Univariate analysis } \\
\hline $\begin{array}{l}\text { CD } 68+\text { macrophage number } \\
(<32 \text { vs }>32)\end{array}$ & $3.638(1.755-7.539)$ & 0.001 & $2.353(1.222-4.531)$ & 0.010 \\
\hline $\begin{array}{l}\text { Tobacco (non smoker vs } \\
\text { smoker) }\end{array}$ & $1.570(0.691-3.568)$ & 0.281 & $1.647(0.728-3.726)$ & 0.231 \\
\hline $\begin{array}{l}\text { Alcohol (non drinker vs } \\
\text { drinker) }\end{array}$ & $1.534(0.763-3.084)$ & 0.230 & $1.392(0.692-2.801)$ & 0.353 \\
\hline Tumor stage (I-II vs III-IV) & $3.017(1.363-6.677)$ & 0.006 & $3.166(1.414-7.089)$ & 0.005 \\
\hline HPV status (HPV- vs HPV+) & $0.517(0.184-1.457)$ & 0.212 & $1.020(0.472-2.205)$ & 0.959 \\
\hline \multicolumn{5}{|l|}{ Multivariate analysis } \\
\hline $\begin{array}{l}\text { CD } 68+\text { macrophage number } \\
(<32 \text { vs }>32)\end{array}$ & $4.210(1.672-10.602)$ & 0.002 & $2.833(1.218-6.589)$ & 0.016 \\
\hline $\begin{array}{l}\text { Tobacco (non smoker vs } \\
\text { smoker) }\end{array}$ & $0.885(0.201-3.899)$ & 0.872 & $1.272(0.348-4.646)$ & 0.716 \\
\hline $\begin{array}{l}\text { Alcohol (non drinker vs } \\
\text { drinker) }\end{array}$ & $1.159(0.360-3.734)$ & 0.805 & $0.640(0.238-1.721)$ & 0.377 \\
\hline Tumor stage (I-II vs III-IV) & $3.622(1.293-10.147)$ & 0.014 & $3.519(1.288-9.616)$ & 0.014 \\
\hline HPV status (HPV-vs HPV+) & $0.306(0.085-1.100)$ & 0.070 & $0.378(0.110-1.303)$ & 0.123 \\
\hline
\end{tabular}

a higher number of these cells in the stromal compartment of advanced-stage tumors (III and IV) (Figure 2A), such as in intra-tumoral regions (Figure 2B). Moreover, when evaluating the stromal compartment, CD68+ macrophage density was significantly different depending on the observed tumor location (Kruskal-Wallis test, $p<0.05$ ). CD68+ macrophage numbers were significantly higher in larynx than in hypopharynx $(p=0.043)$ and oropharynx carcinomas $(p=0.023$ ) (Figure 2C). No differences in $\mathrm{CD} 68+$ macrophage numbers were observed between the several locations studied when looking at the intratumoral compartment. Concerning HPV status, CD68+ macrophage numbers were statistically higher in the intra-tumoral compartment of $\mathrm{HPV}+/ \mathrm{p} 16+$ tumors than in $\mathrm{HPV}+/ \mathrm{p} 16-$ and HPV- tumors $(p=0.003)$ (Figure 2D).

\section{CD68+ macrophage infiltration increases during HNSCC progression}

We evaluated CD68+ macrophage numbers in 10 cases of tumor-free peri-tumoral epithelia (TFE), 43 cases of low-grade dysplasia (LGD), 45 cases of high-grade dysplasia (HGD) and 110 cases of carcinoma (CA). We observed increased CD68+ macrophage recruitment during disease progression (from TFE to CA) in both the intraepithelial compartment and in the stromal compartment (Figure 3A). Kruskal-Wallis test revealed that this increase was statistically significant in both compartments $(p<0.001)$ (Figures 3B-3C). In addition, we noticed a higher number of CD68+ macrophages in stromal regions (max. 240 cells per field, 400X magnification) than in epithelial regions (max. 110 cells per field) (Figures 3B-3C). In the intra-epithelial compartment, CD68+ macrophage numbers were significantly different between TFE and LGD (post hoc test, $p=0.005$ ), TFE and HGD $(p<0.001)$, and TFE and CA $(p<0.001)$. Moreover, there was a statistically significant difference between LGD and HGD $(p=0.003)$ and LGD and CA $(p=0.004)$ (Figure 3B). In the stromal compartment, we noticed a significant difference between dysplasia (low-grade and high-grade) and carcinoma (post hoc test, $p<0.001$ ) (Figure $3 \mathrm{C}$ ). Concerning HPV status, CD68+ macrophage numbers increased during disease progression, despite HPV status (Figure 4) but was significantly higher in the intra-tumoral compartment of $\mathrm{HPV}+/ \mathrm{p} 16+$ tumors than in $\mathrm{HPV}+/ \mathrm{p} 16-$ and HPV- tumors $(p=0.003)$ (Figure 5A). This difference was not statistically significant in the stromal compartment (Figure 5B).

\section{CD68+ macrophage recruitment is decreased by HPV16-E6 oncoprotein}

To determine the implication of HPV16-E6 and E7 oncoproteins in $\mathrm{CD} 68+$ macrophage recruitment, we inoculated SCC-VII cells transfected with HPV16-E6, $-\mathrm{E} 7,-\mathrm{E} 6 / \mathrm{E} 7$ or not (control, CT) in female $\mathrm{C} 3 \mathrm{H} / \mathrm{HeN}$ mice. First, CD68+ macrophage numbers were evaluated in tumor samples derived from the four groups of mice 
and appeared to be significantly different (Kruskal-Wallis test, $p=0.006$ ). Indeed, we observed a higher number of CD68+ macrophages in the control group compared to the E6 group ( $p=0.012)$, and a significantly higher CD68+ macrophage number in the E7 and E6/E7 groups compared to E6 alone ( $p=0.029$ and $p<0.001)$ (Supplementary Figure 1). This observation is in accordance with the invasion assay performed by co-culturing the RAW macrophage cell line with SCC-VII-CT, -E6, -E7 and $-\mathrm{E} 6 / \mathrm{E} 7$. Indeed, the number of invaded macrophages was higher when exposed to SCC-VII-CT than to -E6, and higher in -E7 and -E6/E7 compared to -E6 alone; however, this observation was not statistically significant $(p=0.085)$ (Supplementary Figure 2).

\section{CD206 immunostaining in SCC-VII E6/E7 mouse tumors}

After staining the entire macrophage population in SCC-VII tumors, we wanted to distinguish the proportion of M1 and M2 macrophages in these tumors. We used CD206 immunostaining to detect M2 (TAMs) macrophages. We observed good CD206 staining in mouse lung tissue (positive control tissue), but no CD206+
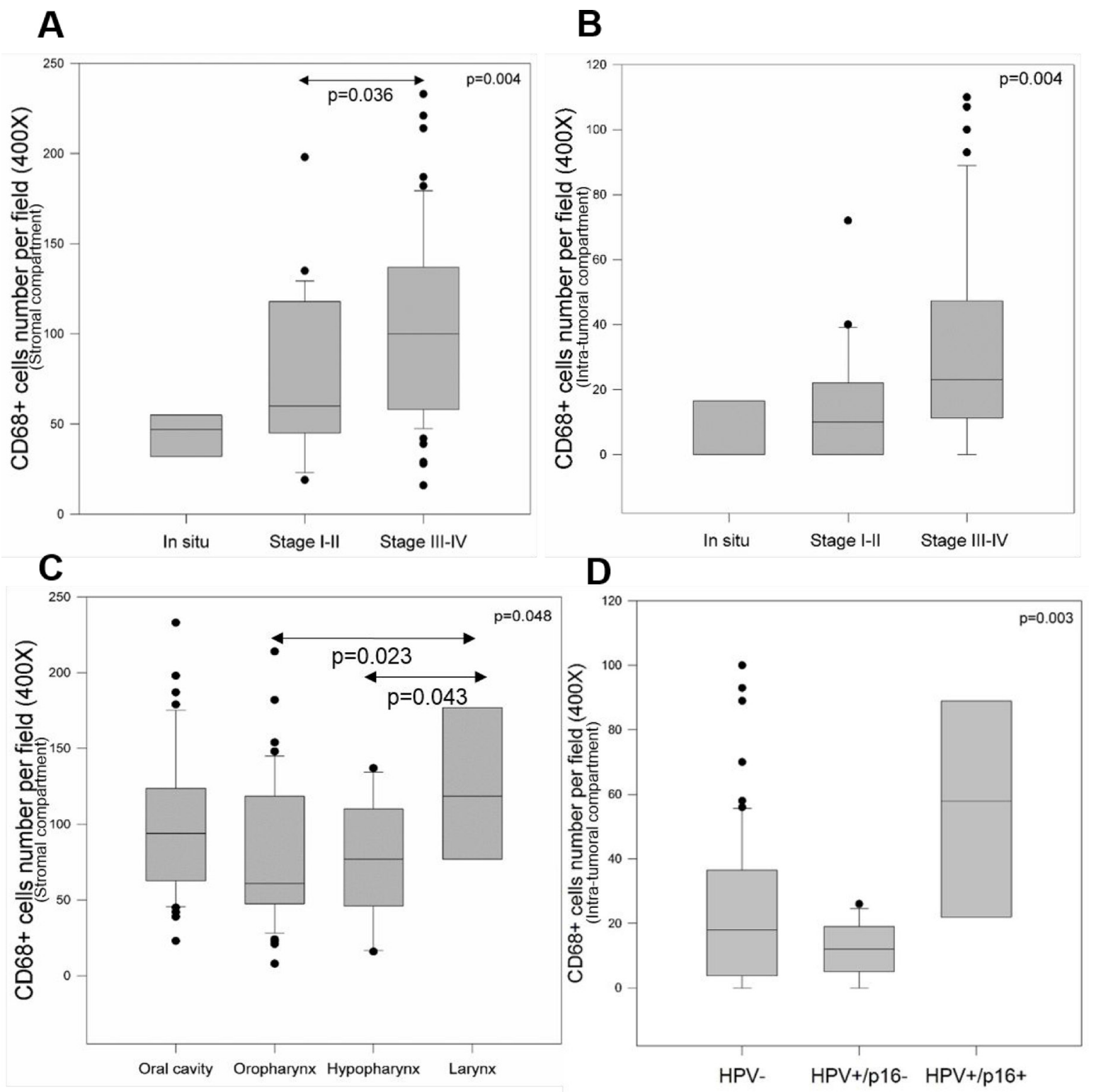

Figure 2: CD68+ macrophages are correlated with tumor stage, tumor location and HPV status. Evaluation of CD68+ macrophage number in stromal regions (A) and intra-tumoral regions (B) of in situ lesions, stages I-II tumors and stages III-IV tumors (Kruskal-Wallis test, $p=0.004)$. Evaluation of CD68+ macrophage number in the stromal compartment of tumors of the oral cavity, oropharynx, hypopharynx and larynx (Kruskal-Wallis test, $p=0.048)(\mathbf{C})$. Evaluation of CD68+ macrophage number in the intra-tumoral compartment of HPV, HPV+/p16- and HPV+/p16+ tumors (Kruskal-Wallis test, $p=0.003)(\mathbf{D})$. 
macrophages were detected in SCC-VII CT, -E6, -E7 or -E6/E7 tumors (Supplementary Figure 3).

\section{DISCUSSION}

Macrophages represent critical mediators of inflammatory processes induced by innate and adaptive immune systems. They also play an important role in HNSCC progression, whether by promoting it (for M2 tumor-associated macrophages) or by repressing it (for M1 macrophages). The main marker used in immunohistochemistry to detect both M1 and M2 macrophages is CD68 [30, 31]. In this study, we showed that CD68+ macrophage infiltration significantly increases during head and neck tumor progression, both in the intraepithelial compartment and in the stromal compartment. Indeed, we observed a significantly higher density of CD68+ macrophages in carcinoma compared to dysplasia (high-grade and low-grade) and to tumor-free peri-tumoral epithelia. If we consider the stromal compartment, we
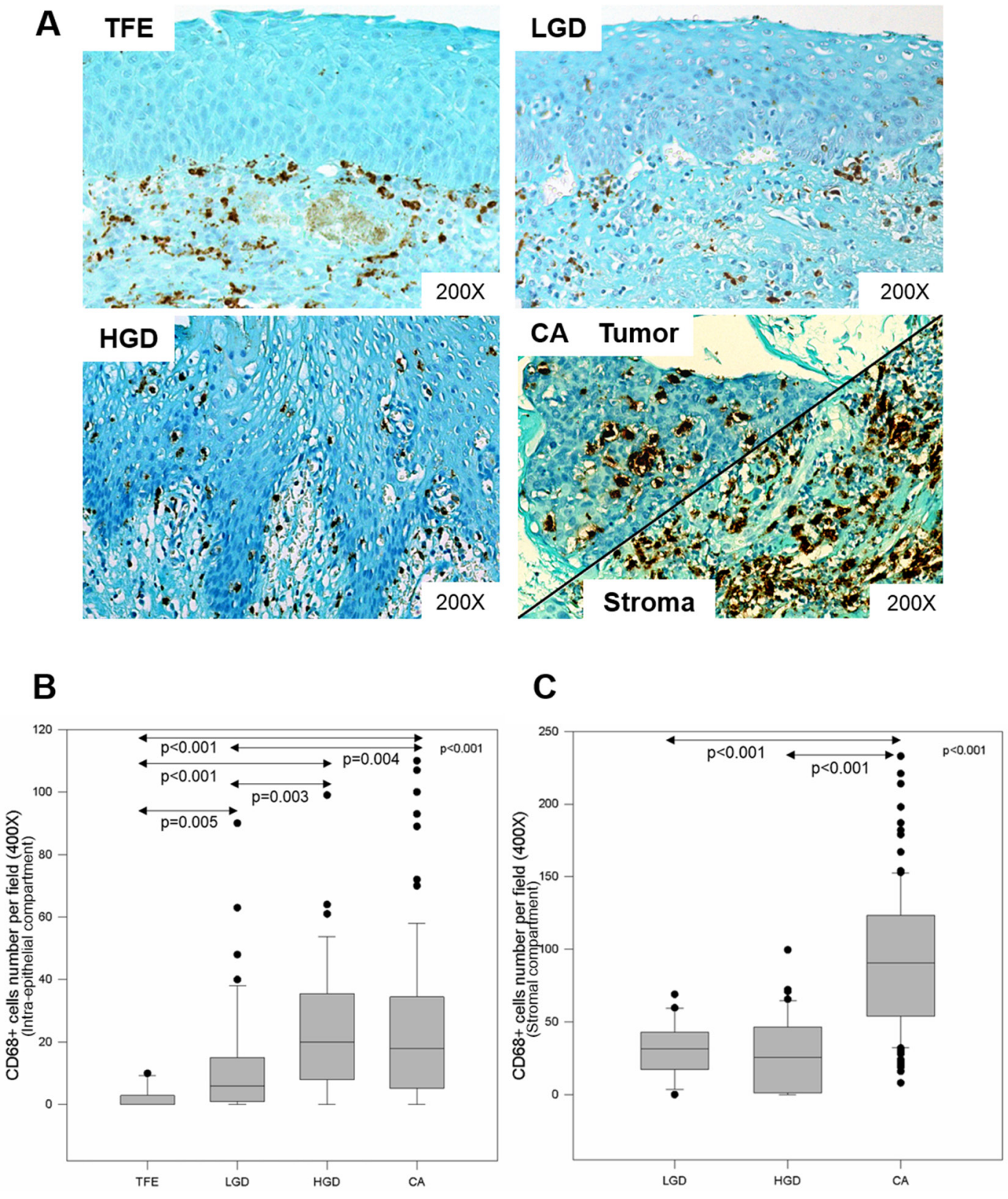

Figure 3: CD68+ macrophage infiltration increases during HNSCC progression. Immunohistochemical representation of CD68 during HNSCC progression in epithelial and stromal compartments, from tumor-free peri-tumoral region, low-grade dysplasia, high-grade dysplasia to carcinoma region (A). CD68+ macrophage number during tumor progression in the intra-tumoral compartment (Kruskal-Wallis test, $p<0.001$ ) (B) and the stromal compartment (Kruskal-Wallis test, $p<0.001)(\mathbf{C})$. 
observed a higher number of CD68+ macrophages in the stromal region of carcinoma than in the stromal region under dysplasia, and these differences were statistically significant. These results are in accordance with several studies demonstrating significantly increasing CD68+ macrophage infiltration from oral normal mucosa to oral squamous cell carcinoma (OSCC), suggesting that CD68 immunostaining could be an important diagnostic and prognostic factor for OSCCs [30, 32, 33].
Our study also showed correlations between CD68+ macrophage infiltration and some clinical data. Indeed, we found a significant correlation between $\mathrm{CD} 68+$ macrophage number and tumor stage, with the density of CD68+ macrophages being higher in advanced stages (III and IV) in both intra-tumoral and stromal compartments. This was also observed in oropharyngeal squamous cell carcinomas and cervical carcinomas, where CD68+ macrophage infiltration was independently associated with
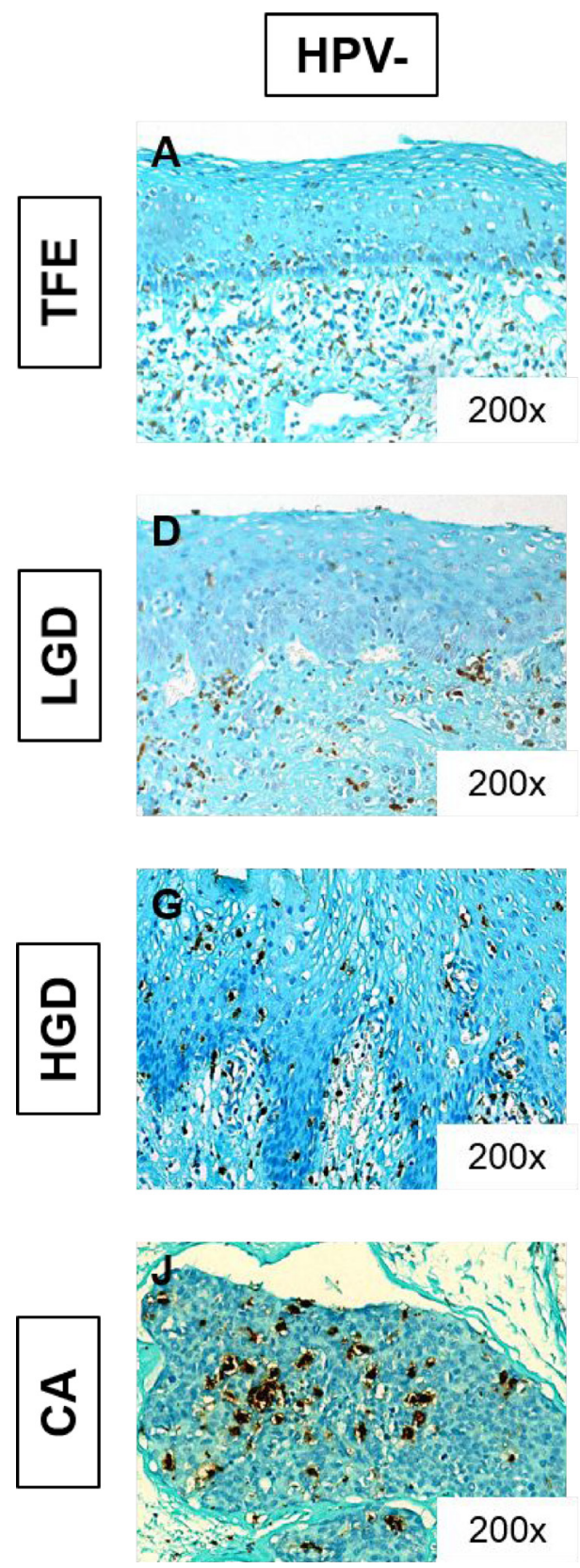
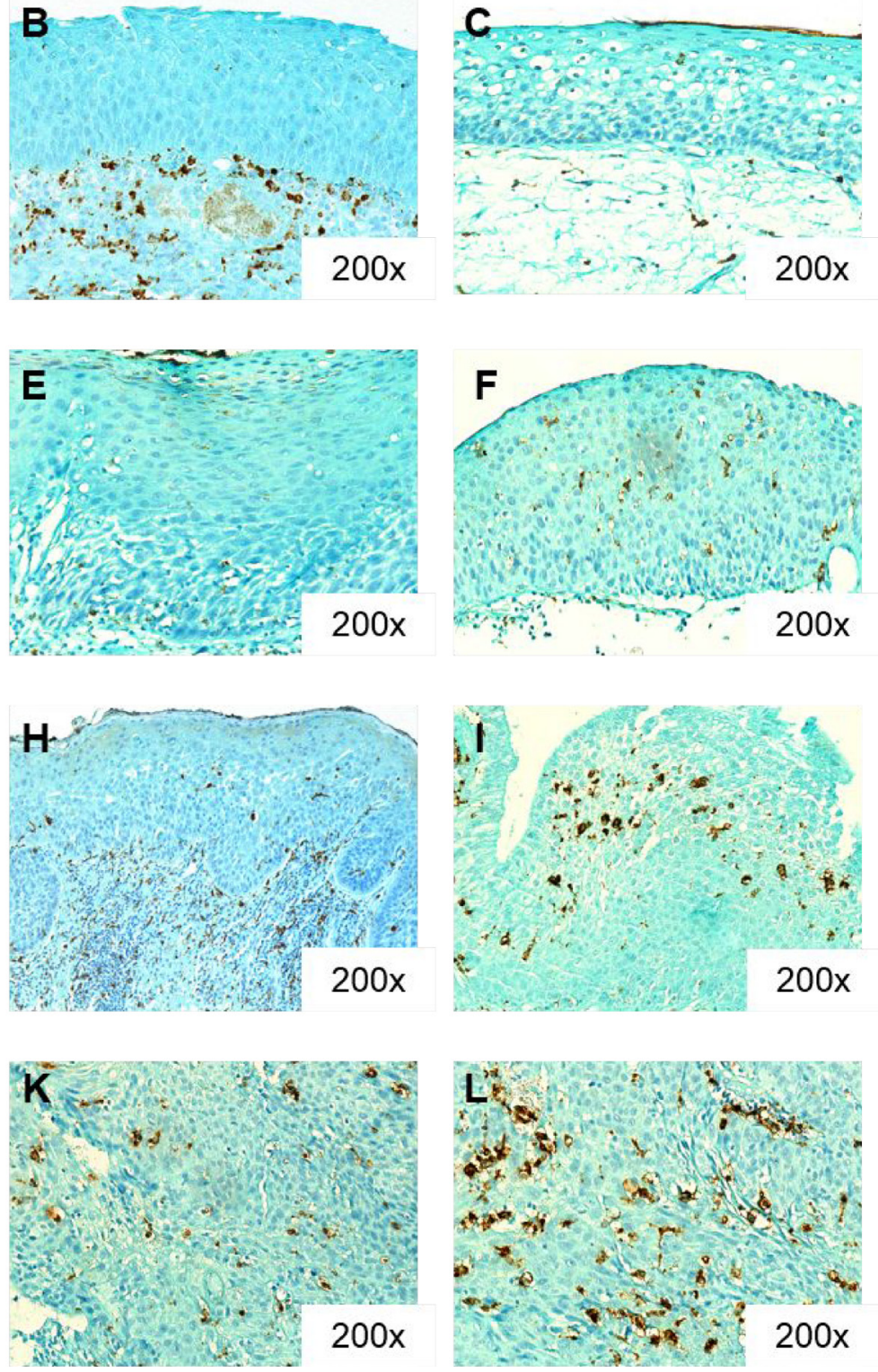

Figure 4: CD68+ macrophage infiltration increases during HNSCC progression, despite HPV status. Immunohistochemical representation of CD68 in tumor-free peritumoral epithelium (TFE) (A, B, C), low-grade dysplasia (LGD) (D, E, F), high-grade dysplasia (HGD), (G, H, I) and carcinoma (CA) (J,K,L) from HPV- patients (A, D, G, J), HPV+/p16- patients (B, E, H, K) and HPV+/p16+ patients (C, F, I, L). 

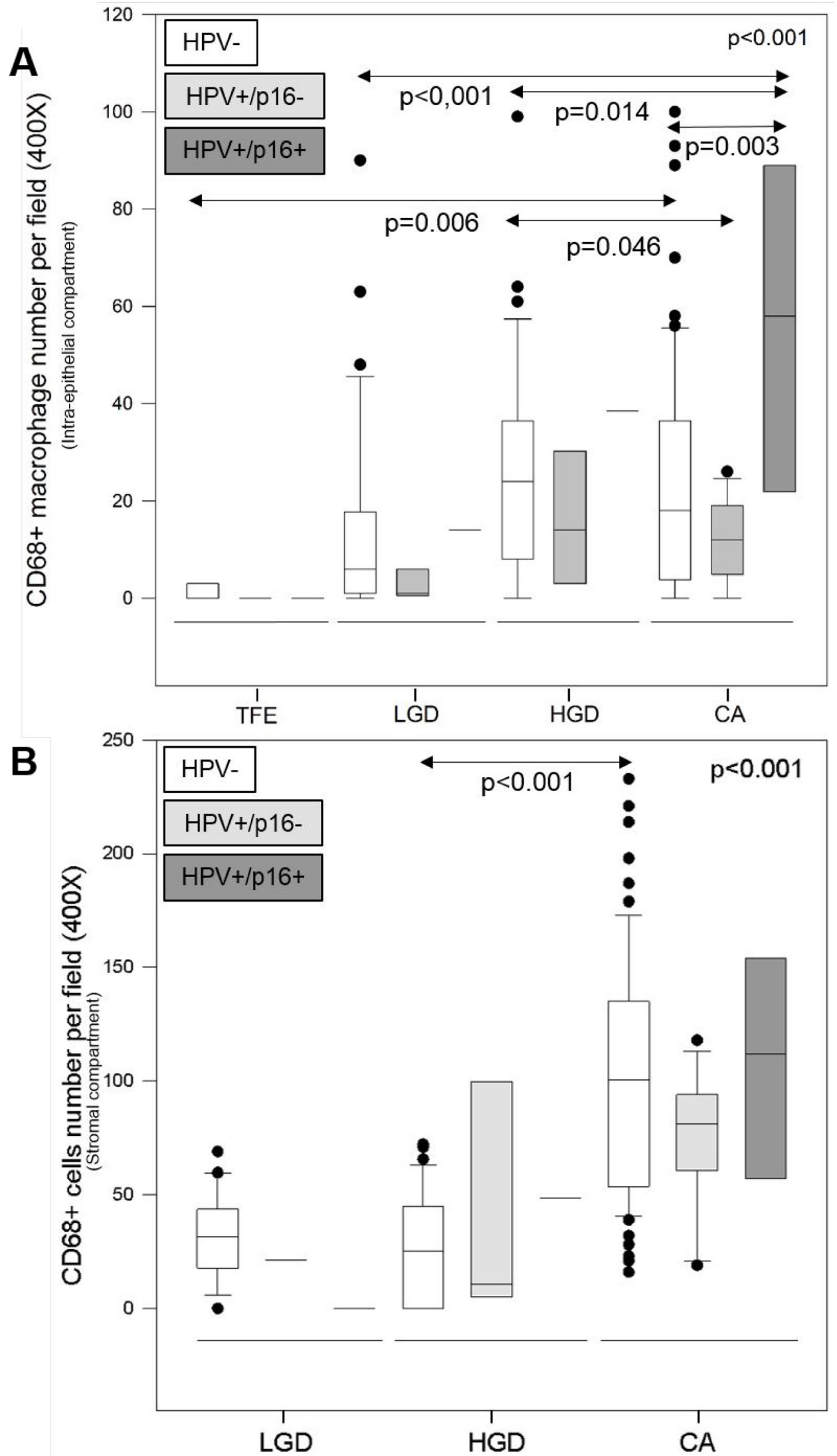

Figure 5: CD68+ macrophage infiltration is higher in HPV+/p16+ HNSCC. CD68+ macrophage number during tumor progression according to HPV status in the intra-tumoral compartment (Kruskal-Wallis test, $p=0.003$ ) (A) and the stromal compartment (Kruskal-Wallis test, $p<0.001$ ) (B). 
Table 2: Characteristics of patient population used in this study

\begin{tabular}{|c|c|c|c|}
\hline Variables & Number of cases $(N=110)$ & $\begin{array}{c}p \text { value CD68 in intra-tumoral } \\
\text { compartment }\end{array}$ & $\begin{array}{c}p \text { value CD68 in stromal } \\
\text { compartment }\end{array}$ \\
\hline Age (years) & & 0.489 & 0.461 \\
\hline Median & 58 & & \\
\hline Range & $37-88$ & & \\
\hline Gender & & 0.122 & 0.359 \\
\hline Male & 81 & & \\
\hline Female & 29 & & \\
\hline Anatomic site & & 0.297 & 0.048 \\
\hline Oral cavity & 42 & & \\
\hline Oropharynx & 48 & & \\
\hline Larynx & 8 & & \\
\hline Hypopharynx & 11 & & \\
\hline Nasopharynx & 1 & & \\
\hline $\begin{array}{l}\text { Histological grade } \\
\text { (differentiation) }\end{array}$ & & 0.920 & 0.887 \\
\hline Well & 52 & & \\
\hline Moderate & 43 & & \\
\hline Poor & 3 & & \\
\hline Unknown & 12 & & \\
\hline Tumor stage & & 0.004 & 0.004 \\
\hline In situ & 2 & & \\
\hline I-II & 37 & & \\
\hline III-IV & 56 & & \\
\hline Unknown & 15 & & \\
\hline \multicolumn{4}{|l|}{ Risk factors } \\
\hline Alcohol & & 0.652 & 0.120 \\
\hline Drinker & 45 & & \\
\hline Non drinker & 33 & & \\
\hline Unknown & 32 & & \\
\hline Tobacco & & 0.213 & 0.675 \\
\hline Smokers & 58 & & \\
\hline Non smokers & 30 & & \\
\hline Unknown & 22 & & \\
\hline HPV status & & 0.445 & 0.197 \\
\hline Negative & 82 & & \\
\hline Positive (p16-) & 12 & & \\
\hline Positive (p16+) & 6 & & \\
\hline Positive (p16 unknown) & 10 & & \\
\hline $\begin{array}{l}\text { Recurrence-free survival } \\
\text { (RFS) }\end{array}$ & & 0.001 & 0.679 \\
\hline Yes & 32 & & \\
\hline None & 52 & & \\
\hline Unknown & 26 & & \\
\hline Overall survival (OS) & & 0.010 & 0.816 \\
\hline Dead & 43 & & \\
\hline Alive & 48 & & \\
\hline Unknown & 19 & & \\
\hline
\end{tabular}


tumor stage [19, 34]. Moreover, we showed a significant correlation between CD68+ macrophage numbers and tumor location. The density of CD68+ macrophages was significantly higher in the stroma of laryngeal tumors than in the stromal compartment of hypopharyngeal and oropharyngeal carcinomas. This result is in accordance with the study of Hu et al., which described a difference in CD68+ macrophage infiltration in different locations of oral squamous cell carcinomas [35].

When evaluating the recurrence-free survival rate and the overall survival rate of patients with HNSCCs according to CD68+ macrophage infiltration, our results showed that a high density of $\mathrm{CD} 68+$ macrophages inside the tumor was associated with shorter recurrence-free and overall survival of HNSCC patients. These results are in accordance with other studies demonstrating that high CD68+ macrophage infiltration was associated with shorter overall survival in head and neck squamous cell carcinomas [35-37]. Likewise, multivariate analyses showed that CD68+ macrophage infiltration, as well as tumor stage, had more significant prognostic values than did other classical risk factors, supporting that CD68+ macrophage density in the intra-tumoral compartment of HNSCCs is a strong and independent prognostic factor for HNSCC patients. These observations could be explained by hypothesizing that the macrophage population in HNSCCs is mainly composed of M2 tumor-associated macrophages.

Regarding HPV status, we noticed in the intratumoral compartment that CD68+ macrophage infiltration was higher in transcriptionally active HPV+ HNSCCs than in HPV+/p16- and HPV- HNSCCs. This is consistent with Oguejiofor et al., whose study used immunohistochemistry to demonstrate significantly higher CD68+ macrophage recruitment in the tumor area of $\mathrm{HPV}+$ oropharyngeal squamous cell carcinomas than in HPV- carcinomas [28].

To better understand the involvement of HPV oncoproteins in $\mathrm{CD} 68+$ macrophage recruitment to the tumor, we developed an orthotopic female mouse model injected with squamous cell carcinoma cell lines transfected with HPV16-E6, -E7 or -E6/E7. Our in vivo study showed significantly higher CD68+ macrophage infiltration in SCC-VII-E7+ (median $=0.2$ ) and SCCVII-E6/E7+ $($ median $=0.4)$ than in SCC-VII-E6+ tumors (median $=0)$. The same result was obtained when coculturing macrophages with SCC-VII-E6/E7+ during the invasion assay. Indeed, the number of invaded macrophages appeared to be higher when exposed to E7 and -E6/E7 oncoproteins than to -E6 alone. However, $\mathrm{CD} 68+$ macrophage infiltration and invasion are the highest in the SCC-VII CT mouse tumors and cell line, which would suggest that HPV-negative mouse tumors have higher $\mathrm{CD} 68+$ macrophage recruitment than do tumors transfected with HPV-E6 and -E7 oncoproteins (considered HPV-positive tumors). This observation in mouse tumors does not seem to be in accordance with the results obtained in human tumors, where CD68+ macrophage density was the highest in HPV+ HNSCCs. We could try to explain these conflicting results by comparing the two biological models. First, CD68+ macrophage numbers are much higher in human tumors (max 110) than in mouse tumors ( $\max 3,5)$, highlighting the deeper interaction between stroma and tumor cells in the human model. In fact, mice were injected with a cancer cell suspension that led to the local establishment of a tumor mass, most often composed of agglomerated and necrotic tumor cells that did not invade the neighbor microenvironment. Second, in the human tumor model, the tumors developed spontaneously, tumor cells invaded the local micro-environment and tumor samples were composed of tumor cells interlaced with stroma and blood vessels. Thus, comparing these two biological models is complex because we cannot distinguish the intra-epithelial region from the stromal region in the mouse model. Third, SCC-VII cells injected in the mouse model were only transfected with HPV16-E6 and -E7 oncoproteins, while human HNSCC tumor cells are infected with the full HPV virus, which is composed of six early genes and two late genes [38]. CD68+ macrophage recruitment could be led by another HPV protein than E6 and E7. Indeed, each protein of the virus plays a role in immune system modulation, as detailed by Sasagawa et al. in cervical cancer [39]. Finally, the significantly higher CD68+ macrophage density in SCCVII-E7+ cells compared to SCC-VII-E6+ cells highlights the ability of E7 to interact differently with the immune system than E6. Indeed, our previous study in the same in vivo model of HNSCC demonstrated that FoxP3 T cell infiltration was significantly higher in SCC-VII-E7+ tumors, suggesting that HPV16-E7 oncoprotein might be a key factor in the regulatory $\mathrm{T}$ cell recruitment in mice [26].

In conclusion, we demonstrate for the first time that CD68+ macrophage infiltration increases during head and neck tumor progression, both in the intra-epithelial and in the stromal compartment. Moreover, high CD68+ macrophage density and advanced tumor stage are associated with shorter recurrence-free and overall survival of HNSCC patients. Our study suggested that CD68+ macrophage infiltration is higher in transcriptionally active $\mathrm{HPV}+\mathrm{HNSCC}$ than in HPV- HNSCCs.

\section{MATERIALS AND METHODS}

\section{Population and clinical data}

Formalin-fixed, paraffin-embedded HNSCC specimens were obtained from 110 patients who underwent curative surgery at CHU Sart-Tilman (Liège, Belgium) and EpiCURA Baudour Hospital (Baudour, Belgium) during the years 2001 to 2011. Among these patients, 82 (74\%) were not infected with HPV, $12(11 \%)$ were infected with a transcriptionally non-active HPV (HPV+/p16-) 
and $6(5 \%)$ were infected with a transcriptionally active HPV (HPV+/p16+). Based on tobacco and/or alcohol consumption, patients were classified as smokers and nonsmokers as well as drinkers and non-drinkers at the time of the HNSCC diagnosis (Table 1). This retrospective study was approved by the Institutional Review Board.

\section{DNA extraction}

Formalin-fixed, paraffin-embedded tissue samples were sectioned $(10 \times 5 \mu \mathrm{m})$, deparaffinized and digested with proteinase $\mathrm{K}$ overnight at $56^{\circ} \mathrm{C}$. DNA was extracted using the QIAamp DNA Mini Kit (Qiagen, Benelux, Belgium) as previously described [10].

\section{Detection of HPV by polymerase chain reaction (PCR) amplification}

HPV DNA detection was performed using PCR with GP5+/GP6+ primers (synthesized by Eurogentec, Liege, Belgium) that amplify a consensus region located within the L1 region of the HPV genome, as previously described [10].

\section{Immunohistochemistry}

\section{p16 immunostaining}

All HPV+ samples were immunohistochemically evaluated for p16 expression using the recommended mouse monoclonal antibody (CINtec p16 (clone E6H4), Ventana, Tucson, AZ, USA) and an automated immunostainer (Bond-Max, Leica Microsystems, Wetzlar, Germany), as previously described [24]. The expression of p16 was defined as positive only when both nucleus and cytoplasm were stained and when more than $70 \%$ of tumor cells were stained.

\section{CD68 immunostaining for human tissue}

CD68+ macrophages were detected in human tissues by immunohistochemistry using a CD68 (PG-M1) mouse monoclonal antibody (Dako, Glostrup, Denmark) at a dilution of 1:200. First, the tumor samples were deparaffinized in two xylene baths and then rehydrated in four ethanol baths (decreasing concentrations from 100\% to $70 \%$ ). Next, the samples were immersed in a $4.5 \%$ $\mathrm{H}_{2} \mathrm{O}_{2} /$ methanol bath and finally in distilled water. Epitope retrieval was performed by immersing the samples in EDTA buffer (Klinipath BVBA, Olen, Belgium), followed by heating in a pressure cooker. After epitope retrieval, primary antibody was incubated for one hour at room temperature. Finally, the samples were incubated with a PowerVision Poly-HRP-anti-mouse IgG (Klinipath, Duiven, Holland), and the antigens were visualized via the addition of a solution of 3-3' DAB- $\mathrm{H}_{2} \mathrm{O}_{2}$-EDTA buffer (Liquid DAB, San Ramon, USA) before coloring with Mayer's hemalum (Klinipath, Duiven, Holland) and mounting with a synthetic balm (Thermo Scientific, Pittsburg, PA, USA). To exclude antigen-independent staining, controls for which the incubation step with the primary antibody was omitted were examined. In all cases, these controls were negative. The number of CD68+ macrophages was counted in 5 fields in each area (TFE, LGD, HGD, CA) with an AxioCam MRC5 optical microscope (Zeiss, Hallbergmoos, Germany) at 400X magnification.

\section{CD68 immunostaining for mouse tissue}

CD68+ macrophages were detected in mouse tissues by immunohistochemistry using a CD68 (FA-11) rat monoclonal antibody (ThermoFisher Scientific, Rockford, IL, USA) at a dilution of 1:200. After deparaffinization, epitope retrieval was performed by immersing the sections in citrate buffer (Scytek, UT, USA), followed by heating in a pressure cooker. Thereafter, sections were successively exposed to solutions containing avidin and biotin to avoid false-positive staining reactions resulting from endogenous biotin, followed by casein and antiCD68 incubation overnight. The day after, sections were exposed to the corresponding biotinylated secondary antibody (polyclonal rabbit anti-rat $\mathrm{IgG}$ ) and then to the avidin-biotin-peroxidase complex (ABC kit), both from Vectorlab (Peterborough, UK). Presence of antigen in the sections was visualized by incubation with a chromogenic substrate mixture containing $\mathrm{DAB}$ and $\mathrm{H}_{2} \mathrm{O}_{2}$. Finally, sections were counterstained with luxol fast blue and mounted with a synthetic medium. The number of CD68+ macrophages was counted in 5 fields in each tumor tissue with an AxioCam MRC5 optical microscope at 400× magnification.

\section{CD206 immunostaining for mouse tissue}

CD206+ macrophages were distinguished in mouse tissues by immunohistochemistry using a CD206 (antimannose receptor) rabbit polyclonal antibody (Abcam, Cambridge, United Kingdom) at a dilution of 1:250. The same steps as for CD68 immunostaining were done, but the immersion in $\mathrm{H}_{2} \mathrm{O}_{2}$ /methanol was omitted, as recommended by the manufacturer's protocol. Heatmediated antigen retrieval was performed with citrate buffer, followed by an anti-CD206 incubation at room temperature. Finally, sections were exposed to the corresponding biotinylated secondary antibody (polyclonal donkey anti-rabbit IgG). The last steps of the procedure are similar to those of the CD68 immunostaining.

\section{Cell lines and culture conditions}

Mouse SCCVII cells were transfected with 3 different vectors to express the HPV oncoproteins E6, E7 or both E6/E7 in the Radiation Oncology Department 
at the Université Catholique de Louvain (Prof. Vincent Grégoire), as previously described [26]. The RAW cell line is a murine macrophage cell line that originates from murine blood and is a kind gift from Prof. Alexandre Legrand (University of Mons). This cell line was cultured in DMEM high-glucose medium supplemented with $10 \%$ fetal calf serum (VWR International, Leuven, Belgium), $1 \%$ penicillin-streptomycin and $1 \%$ non-essential amino acids (ThermoFisher Scientific).

\section{In vivo studies}

Animal studies were conducted on 80 female $\mathrm{C} 3 \mathrm{H} /$ HeN mice (Charles River Laboratory, L'Arbresle, France). The animals were maintained and handled in compliance with the guidelines issued by the Belgian Ministry of Trade and Agriculture. A suspension of SCCVII cells transfected for E6, E7 and E6/E7 expression or not (control, CT) was injected in the mylohyoid muscle following a procedure detailed in a previous publication [29]. Animals were monitored for tumor onset and were euthanized when they exhibited a tumor more than $15 \mathrm{~mm}$ diameter or a weight loss of more than $20 \%$.

\section{Involvement of HPV16-E6/E7 oncoproteins in macrophage invasion}

Co-culture of the RAW cell line and SCC-VII-E6, $-\mathrm{E} 7,-\mathrm{E} 6 / \mathrm{E} 7$ and $-\mathrm{CT}$ cells was done in quadruplicate by using Boyden chambers. SCC-VII cell lines were seeded in 12-wells plate $\left(4 \times 10^{4}\right.$ cells per well) and cultured for 72 hours. After that, SCC-VII medium was renewed, and RAW cells were seeded in Boyden chambers $\left(1 \times 10^{5}\right.$ cells per chamber) that were placed in the wells culturing SCCVII cells for 24 hours. The last step consisted of staining the invaded macrophages with crystal violet.

\section{Statistical analyses}

The medians of the independent data groups were compared using nonparametric Mann-Whitney test (2 groups) or Kruskal-Wallis test ( $>2$ groups). When the latter test was significant, the Dunn post hoc test was used to compare each pair of groups (to avoid multiple comparison effects). The optimal cutoff points of the populations were estimated using Cutoff finder web application [40]. Recurrence-free survival and overall survival analyses were performed using Kaplan-Meier curves, and the results were compared with log-rank tests. Univariate and multivariate Cox regression models were applied to calculate the hazard ratio (HR) and 95\% confidence interval $(95 \% \mathrm{CI})$ and to assess the independent contributions of CD68+ macrophages to RFS and OS in the presence of other covariates, including tobacco, alcohol, HPV status and tumor stage. $P$-values $<0.05$ were considered statistically significant. All statistical analyses were performed using SigmaPlot 11.0 (Systat Software,
San Jose, CA, USA) and SPSS 23 (IBM, Chicago, IL, USA).

\section{ACKNOWLEDGMENTS}

We thank Prof. Grégoire for providing us SCCVII-E6, -E7, -E6/E7 and -CT cell lines. We also thank Prof. Legrand for providing RAW macrophages cell line.

\section{CONFLICTS OF INTEREST}

No conflicts of interest.

\section{FUNDING}

Imelda Seminerio is supported by a grant Televie from the Belgian National Found for Scientific Research, F.R.S-FNRS (grant $\mathrm{n}^{\circ}$ 7461017F).

\section{REFERENCES}

1. Jemal A, Bray F, Center MM, Ferlay J, Ward E, Forman D. Global cancer statistics. CA Cancer J Clin. 2011; 61:69-90. https://doi.org/10.3322/caac.20107.

2. GLOBOCAN. World Health Organization, 2012.

3. Gillison ML, Koch WM, Capone RB, Spafford M, Westra WH, Wu L, Zahurak ML, Daniel RW, Viglione M, Symer DE, Shah KV, Sidransky D. Evidence for a causal association between human papillomavirus and a subset of head and neck cancers. J Natl Cancer Inst. 2000; 92:709-20.

4. Kreimer AR, Clifford GM, Boyle P, Franceschi S. Human papillomavirus types in head and neck squamous cell carcinomas worldwide: a systematic review. Cancer Epidemiol Biomarkers Prev. 2005; 14:467-75. https://doi. org/10.1158/1055-9965.EPI-04-0551.

5. Curado MP, Boyle P. Epidemiology of head and neck squamous cell carcinoma not related to tobacco or alcohol. Curr Opin Oncol. 2013; 25:229-34. https://doi.org/10.1097/ CCO.0b013e32835ff48c.

6. Young D, Xiao CC, Murphy B, Moore M, Fakhry C, Day TA. Increase in head and neck cancer in younger patients due to human papillomavirus (HPV). Oral Oncol. 2015; 51:727-30. https://doi.org/10.1016/j. oraloncology.2015.03.015.

7. Fakhry C, Westra WH, Li S, Cmelak A, Ridge JA, Pinto H, Forastiere A, Gillison ML. Improved survival of patients with human papillomavirus-positive head and neck squamous cell carcinoma in a prospective clinical trial. J Natl Cancer Inst. 2008; 100:261-9. https://doi.org/10.1093/jnci/djn011.

8. Ang KK, Harris J, Wheeler R, Weber R, Rosenthal DI, Nguyen-Tân PF, Westra WH, Chung CH, Jordan RC, Lu C, Kim H, Axelrod R, Silverman CC, et al. Human papillomavirus and survival of patients with oropharyngeal cancer. 
N Engl J Med. 2010; 363:24-35. https://doi.org/10.1056/ NEJMoa0912217.

9. Duray A, Descamps G, Decaestecker C, Remmelink M, Sirtaine N, Lechien J, Ernoux-Neufcoeur P, Bletard N, Somja J, Depuydt CE, Delvenne P, Saussez S. Human papillomavirus DNA strongly correlates with a poorer prognosis in oral cavity carcinoma. Laryngoscope. 2012; 122:155865. https://doi.org/10.1002/lary.23298.

10. Duray A, Descamps G, Decaestecker C, Sirtaine N, Gilles A, Khalifé M, Chantrain G, Depuydt CE, Delvenne P, Saussez S. Human papillomavirus predicts the outcome following concomitant chemoradiotherapy in patients with head and neck squamous cell carcinomas. Oncol Rep. 2013; 30:371-6. https://doi.org/10.3892/or.2013.2415.

11. Descamps G, Karaca Y, Lechien JR, Kindt N, Decaestecker C, Remmelink M, Larsimont D, Andry G, Hassid S, Rodriguez A, Khalife M, Journe F, Saussez S. Classical risk factors, but not HPV status, predict survival after chemoradiotherapy in advanced head and neck cancer patients. J Cancer Res Clin Oncol. 2016; 142:2185-96. https://doi. org/10.1007/s00432-016-2203-7.

12. Whiteside TL. Immunobiology of head and neck cancer. Cancer Metastasis Rev. 2005; 24:95-105. https://doi. org/10.1007/s10555-005-5050-6.

13. Duray A, Demoulin S, Hubert P, Delvenne P, Saussez S. Immune suppression in head and neck cancers: a review. Clin Dev Immunol. 2010; 2010:701657. https://doi. org/10.1155/2010/701657.

14. Lewis CE, Pollard JW. Distinct role of macrophages in different tumor microenvironments. Cancer Res. 2006; 66:605-12. https://doi.org/10.1158/0008-5472. CAN-05-4005.

15. Lamagna C, Aurrand-Lions M, Imhof BA. Dual role of macrophages in tumor growth and angiogenesis. J Leukoc Biol. 2006; 80:705-13. https://doi.org/10.1189/jlb.1105656.

16. Siveen KS, Kuttan G. Role of macrophages in tumour progression. Immunol Lett. 2009; 123:97-102. https://doi. org/10.1016/j.imlet.2009.02.011.

17. Coffelt SB, Hughes R, Lewis CE. Tumor-associated macrophages: effectors of angiogenesis and tumor progression. Biochim Biophys Acta. 2009; 1796:11-8. https://doi. org/10.1016/j.bbcan.2009.02.004.

18. Qian BZ, Pollard JW. Macrophage diversity enhances tumor progression and metastasis. Cell. 2010; 141:39-51. https:// doi.org/10.1016/j.cell.2010.03.014.

19. Hammes LS, Tekmal RR, Naud P, Edelweiss MI, Kirma N, Valente PT, Syrjänen KJ, Cunha-Filho JS. Macrophages, inflammation and risk of cervical intraepithelial neoplasia (CIN) progression-clinicopathological correlation. Gynecol Oncol. 2007; 105:157-65. https://doi.org/10.1016/j. ygyno.2006.11.023.

20. Mazibrada J, Rittà M, Mondini M, De Andrea M, Azzimonti B, Borgogna C, Ciotti M, Orlando A, Surico N, Chiusa L, Landolfo S, Gariglio M. Interaction between inflammation and angiogenesis during different stages of cervical carcinogenesis. Gynecol Oncol. 2008; 108:112-20. https://doi. org/10.1016/j.ygyno.2007.08.095.

21. van Esch EM, van Poelgeest MI, Trimbos JB, Fleuren GJ, Jordanova ES, van der Burg SH. Intraepithelial macrophage infiltration is related to a high number of regulatory $\mathrm{T}$ cells and promotes a progressive course of HPV-induced vulvar neoplasia. Int J Cancer. 2015; 136:E85-94. https://doi. org/10.1002/ijc.29173.

22. de Vos van Steenwijk PJ, Ramwadhdoebe TH, Goedemans R, Doorduijn EM, van Ham JJ, Gorter A, van Hall T, Kuijjer ML, van Poelgeest MI, van der Burg SH, Jordanova ES. Tumor-infiltrating CD14-positive myeloid cells and CD8positive T-cells prolong survival in patients with cervical carcinoma. Int J Cancer. 2013; 133:2884-94. https://doi. org/10.1002/ijc.28309.

23. Li C, Shintani S, Terakado N, Nakashiro K, Hamakawa H. Infiltration of tumor-associated macrophages in human oral squamous cell carcinoma. Oncol Rep. 2002; 9:1219-23.

24. Kindt N, Descamps G, Seminerio I, Bellier J, Lechien JR, Pottier C, Larsimont D, Journé F, Delvenne P, Saussez S. Langerhans cell number is a strong and independent prognostic factor for head and neck squamous cell carcinomas. Oral Oncol. 2016; 62:1-10. https://doi.org/10.1016/j. oraloncology.2016.08.016.

25. Andersen AS, Koldjaer Sølling AS, Ovesen T, Rusan M. The interplay between HPV and host immunity in head and neck squamous cell carcinoma. Int J Cancer. 2014; 134:2755-63. https://doi.org/10.1002/ijc.28411.

26. Kindt N, Descamps G, Seminerio I, Bellier J, Lechien JR, Mat Q, Pottier C, Delvenne P, Journé F, Saussez S. High stromal Foxp3-positive T cell number combined to tumor stage improved prognosis in head and neck squamous cell carcinoma. Oral Oncol. 2017; 67:183-191. https://doi. org/10.1016/j.oraloncology.2017.02.023.

27. Lepique AP, Daghastanli KR, Cuccovia IM, Villa LL. HPV16 tumor associated macrophages suppress antitumor T cell responses. Clin Cancer Res. 2009; 15:4391-400. https://doi.org/10.1158/1078-0432.CCR-09-0489.

28. Oguejiofor K, Galletta-Williams H, Dovedi SJ, Roberts DL, Stern PL, West CM. Distinct patterns of infiltrating CD8+ T cells in HPV+ and CD68 macrophages in HPVoropharyngeal squamous cell carcinomas are associated with better clinical outcome but PD-L1 expression is not prognostic. Oncotarget. 2017; 8:14416-14427. https://doi. org/10.18632/oncotarget.14796.

29. Kindt N, Preillon J, Kaltner H, Gabius HJ, Chevalier D, Rodriguez A, Johnson BD, Megalizzi V, Decaestecker C, Laurent G, Saussez S. Macrophage migration inhibitory factor in head and neck squamous cell carcinoma: clinical and experimental studies. J Cancer Res Clin Oncol. 2013; 139:727-37. https://doi.org/10.1007/s00432-013-1375-7.

30. Lu CF, Huang CS, Tjiu JW, Chiang CP. Infiltrating macrophage count: a significant predictor for the progression and prognosis of oral squamous cell carcinomas in Taiwan. 
Head Neck. 2010; 32:18-25. https://doi.org/10.1002/ hed.21138.

31. Kurahara H, Shinchi H, Mataki Y, Maemura K, Noma H, Kubo F, Sakoda M, Ueno S, Natsugoe S, Takao S. Significance of M2-polarized tumor-associated macrophage in pancreatic cancer. J Surg Res. 2011; 167:e211-9. https:// doi.org/10.1016/j.jss.2009.05.026.

32. El-Rouby DH. Association of macrophages with angiogenesis in oral verrucous and squamous cell carcinomas. J Oral Pathol Med. 2010; 39:559-64. https://doi. org/10.1111/j.1600-0714.2010.00879.x.

33. He KF, Zhang L, Huang CF, Ma SR, Wang YF, Wang WM, Zhao ZL, Liu B, Zhao YF, Zhang WF, Sun ZJ. CD163+ tumor-associated macrophages correlated with poor prognosis and cancer stem cells in oral squamous cell carcinoma. BioMed Res Int. 2014; 2014:838632. https://doi. org/10.1155/2014/838632.

34. Marcus B, Arenberg D, Lee J, Kleer C, Chepeha DB, Schmalbach CE, Islam M, Paul S, Pan Q, Hanash S, Kuick R, Merajver SD, Teknos TN. Prognostic factors in oral cavity and oropharyngeal squamous cell carcinoma. Cancer. 2004; 101:2779-87. https://doi.org/10.1002/cncr.20701.

35. Hu Y, He MY, Zhu LF, Yang CC, Zhou ML, Wang Q, Zhang W, Zheng YY, Wang DM, Xu ZQ, Wu YN, Liu LK. Tumorassociated macrophages correlate with the clinicopathological features and poor outcomes via inducing epithelial to mesenchymal transition in oral squamous cell carcinoma. J Exp Clin Cancer Res. 2016; 35:12. https://doi.org/10.1186/ s13046-015-0281-z.
36. Rittà M, De Andrea M, Mondini M, Mazibrada J, Giordano C, Pecorari G, Garzaro M, Landolfo V, Schena M, Chiusa L, Landolfo S. Cell cycle and viral and immunologic profiles of head and neck squamous cell carcinoma as predictable variables of tumor progression. Head Neck. 2009; 31:318-27. https://doi.org/10.1002/hed.20977.

37. Weber M, Büttner-Herold M, Hyckel P, Moebius P, Distel L, Ries J, Amann K, Neukam FW, Wehrhan F. Small oral squamous cell carcinomas with nodal lymphogenic metastasis show increased infiltration of M2 polarized macrophages-an immunohistochemical analysis. J Craniomaxillofac Surg. 2014; 42:1087-94. https://doi.org/10.1016/j.jcms. 2014.01.035.

38. Doorbar J, Quint W, Banks L, Bravo IG, Stoler M, Broker TR, Stanley MA. The biology and life-cycle of human papillomaviruses. Vaccine. 2012; 30:F55-70. https://doi. org/10.1016/j.vaccine.2012.06.083.

39. Sasagawa T, Takagi H, Makinoda S. Immune responses against human papillomavirus (HPV) infection and evasion of host defense in cervical cancer. J Infect Chemother. 2012; 18:807-15. https://doi.org/10.1007/s10156-012-0485-5.

40. Budczies J, Klauschen F, Sinn BV, Györffy B, Schmitt WD, Darb-Esfahani S, Denkert C. Cutoff Finder: a comprehensive and straightforward Web application enabling rapid biomarker cutoff optimization. PLoS One. 2012; 7:e51862. 